\title{
A model for turbulent dissipation rate in a constant pressure boundary layer
}

\author{
$\mathrm{J} \mathrm{DEY}^{1}$ and P PHANI KUMAR ${ }^{2, *}$ \\ ${ }^{1}$ Faculty, Department of Aerospace Engineering, Jain University, Bangalore 562112, India \\ ${ }^{2}$ Department of Aerospace Engineering, Indian Institute of Science, Bangalore 560012, India \\ e-mail: jd@aero.iisc.ernet.in; phani2565@gmail.com
}

MS received 12 October 2014; accepted 30 October 2015

\begin{abstract}
Estimation of the turbulent dissipation rate in a boundary layer is a very involved process. Experimental determination of either the dissipation rate or the Taylor microscale, even in isotropic turbulence, which may occur in a portion of the turbulent boundary layer, is known to be a difficult task. For constant pressure boundary layers, a model for the turbulent dissipation rate is proposed here in terms of the local mean flow quantities. Comparable agreement between the estimated Taylor microscale and Kolmogorov length scale with other data in the logarithmic region suggests usefulness of this model in obtaining these quantities experimentally.
\end{abstract}

Keywords. Boundary layers; dissipation rate; Kolmogorov length scale; Taylor microscale.

\section{Introduction}

Estimation of the turbulent dissipation rate, $\epsilon$, of the kinetic energy requires the gradients of velocity fluctuations in a boundary layer. While direct numerical simulation (DNS) (although computationally intensive) provides the turbulent dissipation rate accurately, it is not so easily realizable in experiments [1, 2]. Stanislas et al [1] have very briefly mentioned difficulties in measuring the turbulent dissipation rate. These authors and Herpin et al [3], for example, find the isotropic condition to be a good approximation in the logarithmic region. However, measurement of the Taylor microscale remains a difficult task, as it involves correlation function [1]. Consequently, an appreciation of the Taylor microscale, dissipation rate, etc., is lacking in practice due to complexity involved in estimating these quantities. Segalini et al [2] have proposed a novel means for measuring the Taylor microscale from two hot-wire measurements. Once the Taylor microscale is available, the turbulent dissipation rate can be estimated, at least for isotropic turbulence. Alternatively, if a model for the turbulent dissipation rate is available, then the Taylor microscale can easily be estimated, as proposed in this paper. This model is in terms of easily measurable mean flow quantities and the estimated Taylor microscale and the Kolmogorov length scale are found to be comparable with other data in the logarithmic region of a constant pressure boundary layer.

*For correspondence

\section{Analysis}

A zero-pressure gradient boundary layer over a plate that is placed in a free-stream velocity of $U_{\infty}$ is considered here. The streamwise and the wall-normal distances are denoted by $x$ and $y$, respectively. The mean velocity components in these directions are $u$ and $v$, respectively; $u^{\prime}$ and $v^{\prime}$ are the corresponding fluctuating velocity components; the rootmean-square of $u^{\prime}$ is $u_{\mathrm{rms}}\left(=\sqrt{\overline{\overline{u^{\prime 2}}}}\right)$.

From a balance between the production and the turbulent dissipation rates in the logarithmic region, the turbulent dissipation rate is [4]

$$
\epsilon=\frac{u_{\tau}^{3}}{\kappa y},
$$

where $u_{\tau}$ is the friction velocity, and $\kappa$ is the Karman constant. In arriving at this turbulent dissipation rate, the two conditions used are: (i) $\overline{u^{\prime} v^{\prime}}=u_{\tau}^{2}$, and (ii) $\mathrm{d} u / \mathrm{d} y=$ $u_{\tau} / k y$ from the $\log$ profile,

$$
\frac{u}{u_{\tau}}=\frac{1}{\kappa} \ln \left(\frac{y u_{\tau}}{v}\right)+B,
$$

where $\mathrm{B}$ is constant, and $v$ is the kinematic viscosity; $\overline{u^{\prime} v^{\prime}}$ is the Reynolds shear stress. This classical dissipation rate can also be interpreted, as follows.

From the inner law, $u^{+}=y^{+}$, the wall vorticity is $\Omega_{w}=u_{\tau}^{2} / v$; where $u^{+}=u / u_{\tau}$ and $y^{+}=y u_{\tau} / v$. Under the boundary layer approximation, the spanwise vorticity is $\Omega_{z} \sim \partial u / \partial y$. For the log-profile in Eq. (2), $\Omega_{z} \sim u_{\tau} / \kappa y$. 
Expressing the dissipation rate in Eq. (1) in terms of these quantities, we have

$$
\epsilon=v\left(\frac{u_{\tau}^{2}}{v}\right)\left(\frac{u_{\tau}}{\kappa y}\right) \sim v \Omega_{w} \Omega_{z} .
$$

One can thus interpret that $\mathrm{d} u / \mathrm{d} y$ is weighted by the wall vorticity.

Blair and Bennet [5] consider $u_{\tau}$ and $y$ as 'energy containing' scales near the wall. Assuming a balance between the dissipation and production rates of the turbulent kinetic energy, they take the dissipation rate as

$$
\epsilon \approx \frac{\tau}{\rho} \frac{\partial u}{\partial y} \approx c \frac{u_{\tau}^{3}}{y},
$$

where $\tau$ is the shear stress, $\rho$ is the density, and $c$ is a constant. Since the turbulent dissipation rate mostly depends on $u_{\tau}$ and $y$ scales, a quantity that is $O\left(u_{\tau}\right)$ may also be considered in defining the dissipation rate. Interestingly, we find that the quantity $\left(u^{+} u_{\mathrm{rms}}^{+}\right)$is of-the-order of $u_{\tau}^{2}$ in the logarithmic region as shown in figure 1 , where the variation of $\left(u^{+} u_{\mathrm{rms}}^{+}\right)^{1 / 2}$ in the boundary layer is shown for the DNS data of Schlatter and Örlü [6] and experimental data of DeGraaff and Eaton [7]; Re denotes Reynolds number based on the momentum thickness; $u_{\mathrm{rms}}^{+}=u_{\mathrm{rms}} / u_{\tau}$. It can be seen that the quantity $\left(u^{+} u_{\mathrm{rms}}^{+}\right)^{1 / 2} \sim$ constant in the log-layer; the deviation seen at higher Reynolds number is due to the fact that the outer peak in the distribution of $u_{\mathrm{rms}}$ becomes noticeable at higher Reynolds numbers. That is, $\left(u u_{\mathrm{rms}}\right)^{1 / 2}$ in the log-layer is an approximate measure of the skin-friction; this result is new, to the best of our knowledge. In the near wall region the variation is linear in this $\log -\log$ plot.

Based on the above-mentioned points, we consider the dissipation rate as

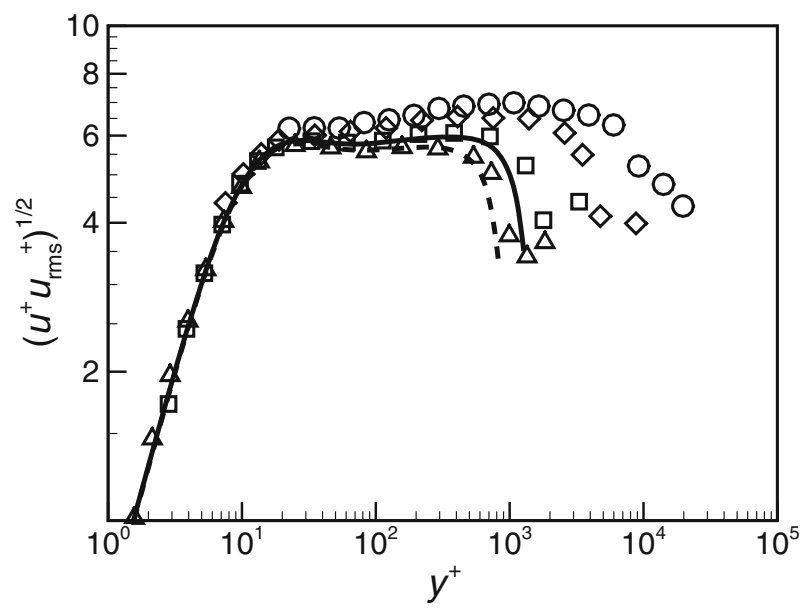

Figure 1. Variation of $\left(u^{+} u_{\mathrm{rms}}^{+}\right)^{1 / 2}$ in the boundary layer. Schlatter and Örlü [6]:,$--- R e=2540 ;-, R e=4060$. DeGraaff and Eaton [7]: $\triangle, R e=2900 ; \square, \quad R e=$ 5160; $\diamond, R e=13310 ; \circ, R e=30850$.

$$
\epsilon \sim v\left(\frac{u_{\tau}^{2}}{v}\right) \frac{\left(u u_{\mathrm{rms}}\right)^{1 / 2}}{y} .
$$

That is, $\left(u u_{\mathrm{rms}}\right)^{1 / 2} / y$, which is $O\left(u_{\tau} / y\right)$, has been weighted by the wall vorticity, as in Eq. (3). For isotropic condition, which is a good approximation in the log-layer $[1,3], u_{\mathrm{rms}}$ is the characteristic velocity scale [4]. The inclusion of $u_{\mathrm{rms}}$ in this model is for this reason. Since $\left(u u_{\mathrm{rms}}\right)^{1 / 2} \sim O\left(u_{\tau}\right)$ in the log-layer, the proposed model for the dissipation rate in Eq. (5) preserves the essence of Eqs. (1) or (4). At the boundary layer edge, $y \rightarrow \delta$ and $u \rightarrow U_{\infty}$. So $\Omega_{z} \sim u_{\tau} / y$ is non-zero. However, $u_{\mathrm{rms}}$ being very small at the boundary layer edge, the quantity $\left(u u_{\mathrm{rms}} / y^{2}\right)^{1 / 2}$ also tends to be small and is caused by the vorticity scale $u_{\mathrm{rms}} / y$ (in terms of the characteristic velocity). In terms of the boundary layer thickness, $\delta$, and $u_{\tau}$, the non-dimensional turbulent dissipation rate is

$$
\frac{\epsilon \delta}{u_{\tau}^{3}}=A \delta^{+} \frac{\left(u^{+} u_{\mathrm{rms}}^{+}\right)^{1 / 2}}{y^{+}} .
$$

Here $\delta^{+}=u_{\tau} \delta / v$. With the constant $A=0.41$, which seems to be equal to the Karman constant, the model turbulent dissipation rate is found to cover a large portion of the boundary layer, including the $\epsilon \sim$ constant region, as shown in figure 2. While this turbulent dissipation model is expected to work well in the logarithmic region, it is a good model for the near wall region, as well. This interesting aspect is attributed to the following. Since $u \sim y$ and $u_{\mathrm{rms}} \sim y, \quad$ as $u^{\prime} \sim y$, near the wall, one expects $\left(u^{+} u_{\mathrm{rms}}^{+}\right)^{1 / 2} \sim y^{+}$there, as shown in figure 1 . The model equation (6) thus yields a constant dissipation rate near the wall. Figure 2 shows that the proposed model for the turbulent dissipation rate, though based on a different consideration, can represent the dissipation rate quite accurately over a large portion of the boundary layer; it is noteworthy that $\mathrm{A}=0.41$ holds good over this region. The foregoing discussion suggests that $\overline{u^{\prime} v^{\prime}}$ and $\left(u u_{\mathrm{rms}}\right)$ may not be totally unrelated.

For constant pressure turbulent boundary layers, the decomposition of the skin-friction coefficient, $C_{f}$, proposed by Fukagata et al [8] is

$$
\begin{aligned}
C_{f}= & \frac{4\left(1-\delta_{1}^{*}\right)}{\operatorname{Re}_{\delta}}+4 \int_{0}^{1}(1-Y)\left(-\overline{u^{\prime} v^{\prime}}\right)_{1} d Y \\
& -2 \int_{0}^{1}(1-Y)^{2}\left(I_{x}+\frac{\partial U}{\partial t}\right) d Y .
\end{aligned}
$$

Here $Y=y / \delta, U=u / U_{\infty},\left(\overline{u^{\prime} v^{\prime}}\right)_{1}=\overline{u^{\prime} v^{\prime}} / U_{\infty}^{2}$, and $\delta_{1}^{*}(=$ $\left.\delta^{*} / \delta\right)$ is the non-dimensional displacement thickness. $I_{x}$ is the inhomogeneous term that contains the inertial part and the streamwise diffusion term, and $\operatorname{Re}_{\delta}\left(=U_{\infty} \delta / v\right)$ is the Reynolds number based on the boundary layer thickness. While the meaning of the first term in the right-hand-side of Eq. (7) is not clear in the case of plane boundary layers, it is 


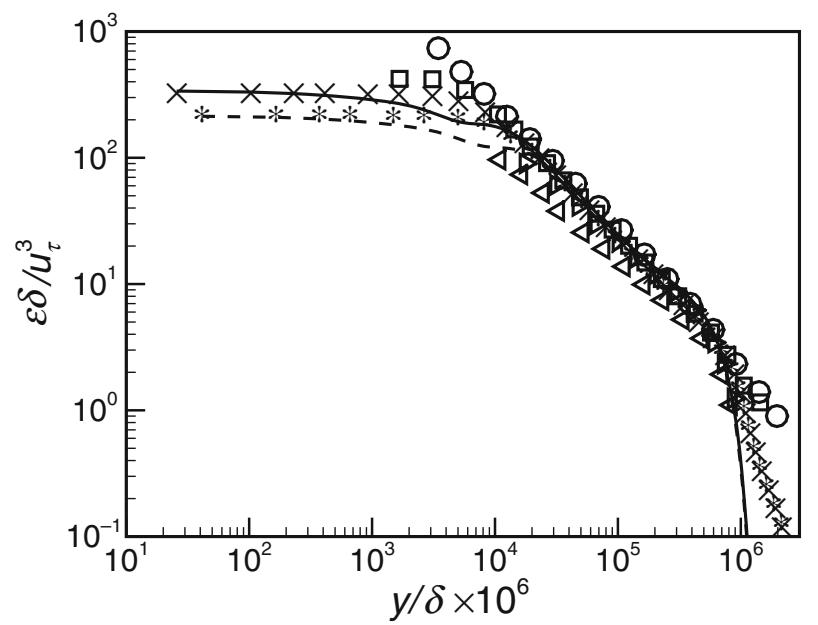

Figure 2. Comparison of the estimated dissipation (symbols) with DNS (lines) and experiments [1] using Eq. (6). Schlatter and Örlü [6]: $*, R e=2540 ; \times, R e=4060$. Stanislas et al [1]: $\triangleright, R e=8200 ; \triangleleft, R e=20800$. Lines and other symbols as in figure 1 .

the laminar skin-friction term in pipe and channel flows. This decomposition of the skin-friction suggests that the Reynolds shear stress, $-\rho\left(\overline{u^{\prime} v^{\prime}}\right)_{1}$, is weighted by the term $(1-Y)$. Now, by definition, the momentum thickness, $\theta$, is

$$
U_{\infty}^{2} \theta=\int_{0}^{\infty} u\left(U_{\infty}-u\right) \mathrm{d} y .
$$

Also, the momentum integral equation for a constant pressure boundary layer is

$$
\frac{\mathrm{d} \theta}{\mathrm{d} x}=\frac{\mathrm{d} \delta}{\mathrm{d} x} \int_{0}^{1} U(1-U) \mathrm{d} Y=\frac{C_{f}}{2} .
$$

Comparing this with Eq. (7), one can infer that $\left(\overline{u^{\prime} v^{\prime}}\right)_{1}$ may have some contribution to the local momentum deficit, $U(1-U)$. Since, for a given Reynolds number, the quantity $(1-Y) / u^{+}\left(U_{\infty}^{+}-u^{+}\right)$is nearly constant in the loglayer, as shown in figure 3 , and $\overline{u^{\prime} v^{\prime}} \sim u_{\tau}^{2}$ there, $U(1-U)$ may be related to $(1-Y) \overline{u^{\prime} v^{\prime}}$ almost directly. As both $\left(\overline{u^{\prime} v^{\prime}}\right)$ and $\left(u u_{\mathrm{rms}}\right)$ being of $O\left(u_{\tau}^{2}\right)$, we find the correlation,

$$
\frac{-\overline{u^{\prime} v^{\prime}}}{\left[u^{+}\left(U_{\infty}^{+}-u^{+}\right)\right]^{1.25}} \sim 4.25 \times 10^{-5} \frac{\left(u^{+} u_{\mathrm{rms}}^{+}\right)}{(1-Y)^{1.038}}
$$

provides a good correspondence between these two terms, as shown in figure 4 . Here again $(1-Y)^{1.038} /\left[u^{+}\left(U_{\infty}^{+}-\right.\right.$ $\left.\left.u^{+}\right)\right]^{1.25}$ is nearly constant in the logarithmic region, as shown in figure 5 .

We now consider the dissipation rate for the isotropic case,

$$
\epsilon=30 v \frac{u_{\mathrm{rms}}^{2}}{\lambda^{2}},
$$

used by Stanislas et al [1]. Using the present dissipation model in Eq. (6), the Taylor microscale that follows for the isotropic case is

$$
\lambda=\left(\frac{30}{A}\right)^{1 / 2}\left(\frac{v y}{u_{\tau}}\right)^{1 / 2} \frac{u_{\mathrm{rms}}}{\left(u_{\tau}^{2} u u_{\mathrm{rms}}\right)^{1 / 4}} .
$$

Noting that $y / u_{\tau}$ is a time scale, one can identify $\left(v y / u_{\tau}\right)^{1 / 2} \sim \sqrt{v t}$ as a length scale; $t$ denotes the time. We may note that the length scale $\left(v y / u_{\tau}\right)^{1 / 2}$ can be arrived at by equating Eq. (1) with (11), as well. Figure 6 shows that the estimated $\lambda^{+}\left(=\lambda u_{\tau} / v\right)$ compares well with the measured data of Stanislas et al [1]; the difference seen for $y^{+} \leq 30$ is because of the isotropic case considered in estimating $\lambda^{+}$. For the log-layer, Alfredsson et al [9] suggest $\lambda^{+}=$ $3.2\left(y^{+}\right)^{1 / 2}$. Figure 6 shows $\lambda^{+}=7.2\left(y^{+}\right)^{1 / 2}$ provides a better fit to the data considered here; this difference could be due to the isotropic relation (Eq. 11) used here. Equation 12 suggests that $\lambda^{+} \sim\left(y^{+}\right)^{1 / 2}\left(u_{\mathrm{rms}}^{+}\right)^{3 / 4} /\left(u^{+}\right)^{1 / 4}$. That is, this differs from Alfredsson et al [9] by the factor $\left(u_{\mathrm{rms}}^{+}\right)^{3 / 4} /\left(u^{+}\right)^{1 / 4}$. However, the variation of $\left(u_{\mathrm{rms}}^{+}\right)^{3 / 4} /\left(u^{+}\right)^{1 / 4}$ being slow in the logarithmic region, compared to $\left(y^{+}\right)^{1 / 2}$, its contribution seems to be small. The data of Segalini et al [2] (taken from their figure 8) shown in figure 6 is multiplied by a factor of 1.6 for it to be closer to other data in this figure. The estimated variation of the Kolmogorov length scale, $\eta=\left(v^{3} / \epsilon\right)^{1 / 4}$, shown in figure 7 is similar to the measured values of Stanislas et al [1] and Herpin et al [3], and is close to $\eta^{+}=\left(\kappa y^{+}\right)^{1 / 4}$ in the loglayer.

A very interesting finding of Herpin et al [3] is that $\Omega_{z}$ scales with the Kolmogorov time scale, $(v \epsilon)^{1 / 2}$, in the loglayer; specifically, they suggest $v \Omega_{z}^{2} \approx \epsilon$. With $u_{\tau}$ and $\left(v y / u_{\tau}\right)^{1 / 2}$ as the characteristic velocity and length scales, we can see in figure 8 that

$$
u_{\tau}\left(\frac{u_{\tau}}{v y}\right)^{1 / 2}\left(\frac{v}{\epsilon}\right)^{1 / 2}=\frac{1}{\left(\epsilon^{+} y^{+}\right)^{1 / 2}} \approx \text { constant },
$$

in the logarithmic region; here DNS [6] and measured [1, 3] data are used for $\varepsilon$. The values estimated using Eq. (6) are also shown for $R e=2540$ [6]. Thus, in terms of $\Omega_{w}$ and $\Omega_{z}$, we have

$$
{ }_{v} \Omega_{w} \Omega_{z} \approx \epsilon,
$$

as in Eq. (3). This is similar to that of Herpin et al [3], except that they consider $\Omega_{z}^{2}$. Now, considering the length 


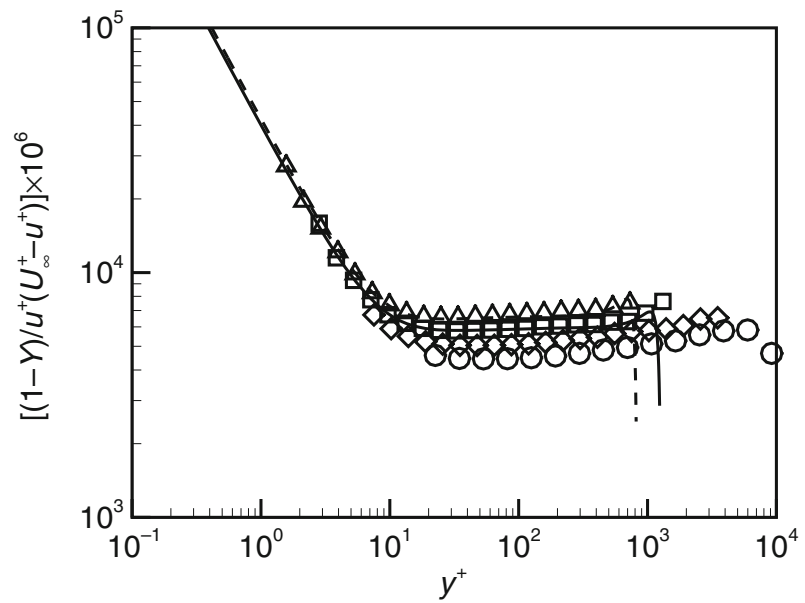

Figure 3. Variation of $(1-Y) / u^{+}\left(U_{\infty}^{+}-u^{+}\right)$in the boundary layer. Lines and other symbols as in figure 1.

(a)

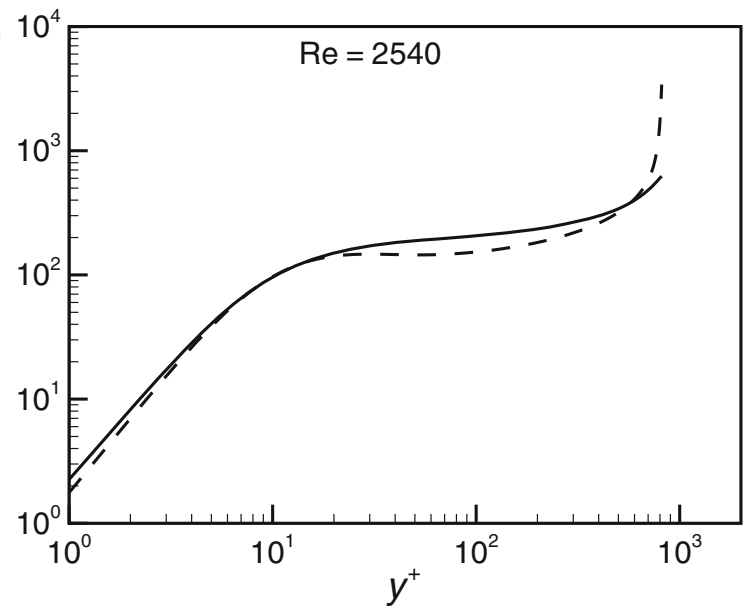

(b)

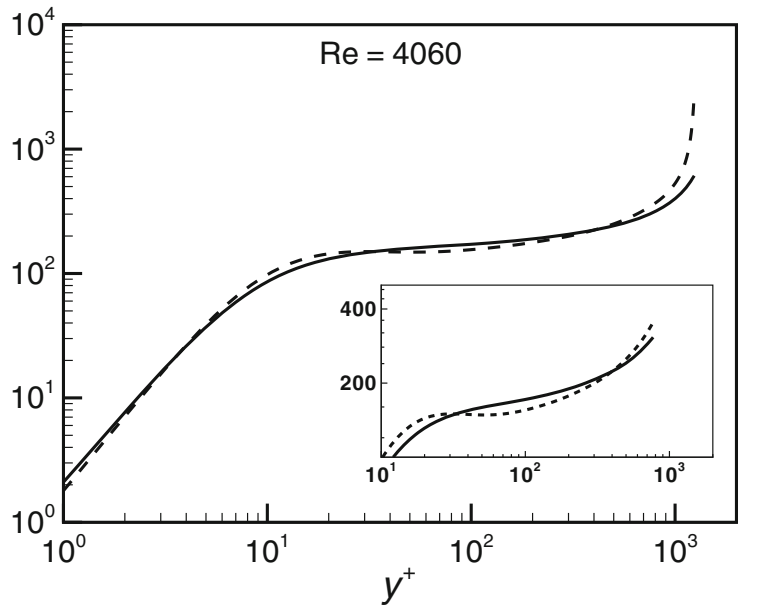

Figure 4. Comparison of $\left[-\left(\overline{u^{\prime} v^{\prime}}\right)^{+} \times 10^{5}\right] /\left[u^{+}\left(U_{\infty}^{+}-u^{+}\right)\right]^{1.25}$ (—) with $4.25 e^{-5}\left[u^{+} u_{\mathrm{rms}}^{+} /(1-Y)^{1.038}\right](---)$. Lines: Schlatter and Örlü [6].

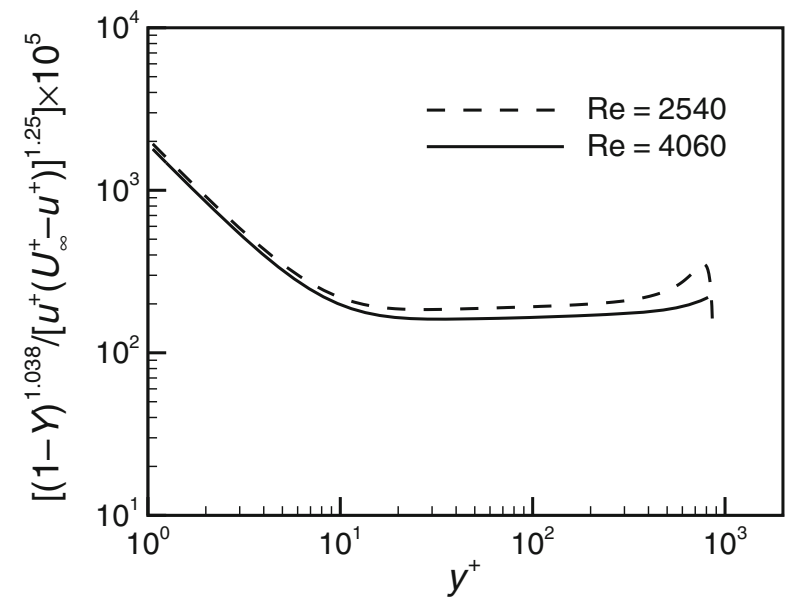

Figure 5. Variation of $(1-Y)^{1.038} /\left[u^{+}\left(U_{\infty}^{+}-u^{+}\right)\right]^{1.25}$ in the boundary layer.

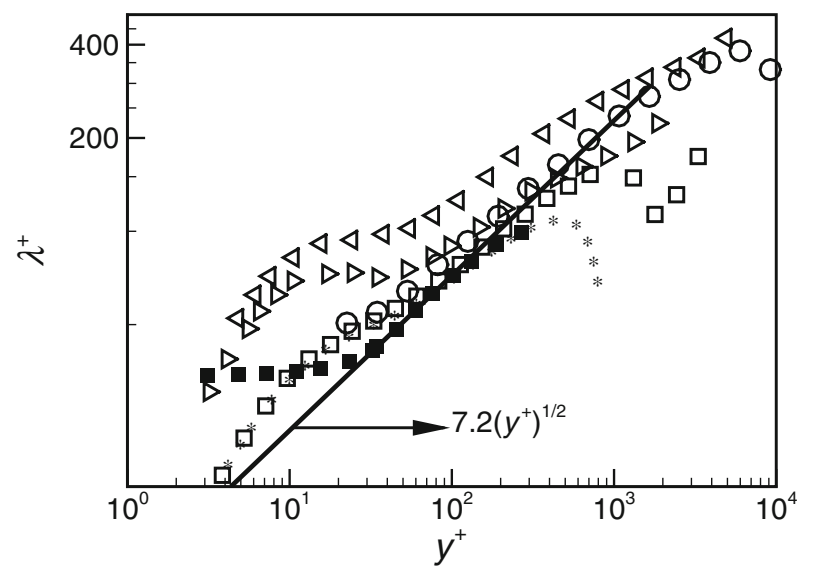

Figure 6. Comparison of the estimated Taylor scale with the data of Stanislas et al [1]. Segalini et al [2]: $\mathbf{\square}, \delta^{+}=1270$. Other symbols as in figures 1 and 2 .

scale $\left(v y / u_{\tau}\right)^{1 / 2}$ and the time scale $y / u_{\tau}$, we have the velocity scale $\left(v u_{\tau} / y\right)^{1 / 2}$. In terms of these length and velocity scales, the scaling of $(v / \epsilon)^{1 / 2}$ is

$$
\left(\frac{v u_{\tau}}{y}\right)^{1 / 2}\left(\frac{u_{\tau}}{v y}\right)^{1 / 2}\left(\frac{v}{\epsilon}\right)^{1 / 2}=\left(\frac{v \Omega_{z}^{2}}{\epsilon}\right)^{1 / 2} .
$$

That is, $\epsilon \approx \nu \Omega_{z}^{2}$ of Herpin et al [3] is arrived at by scaling $(v / \epsilon)^{1 / 2}$ with the time scale $\Omega_{z} \sim\left(u_{\tau} / y\right)$. Thus, $\epsilon \sim v \Omega_{z} \Omega_{w}$ or $\epsilon \sim v \Omega_{z}^{2}$ may depend on the choice of the velocity and length scales; as $\epsilon \sim y^{-1}$ in the logarithmic layer, $\epsilon y=$ constant is expected. Thus the proposed model seems equally good in the scaling of $(v / \epsilon)^{1 / 2}$ with $\Omega_{z}$. It may be noted that, with $\Omega_{z} \sim \partial u / \partial y$, and $\epsilon \sim y^{-1}$ in Eq. (14) leads to $u \sim \ln (y)$. 


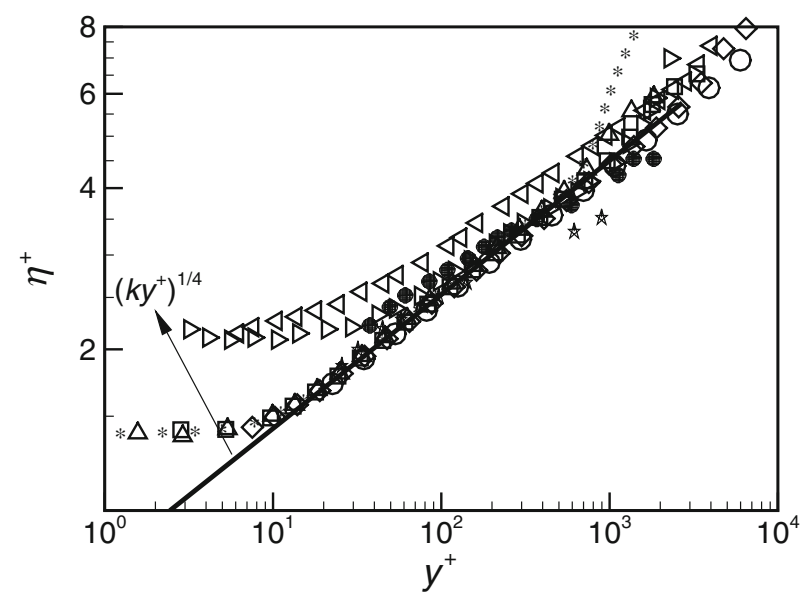

Figure 7. Comparison of the estimated Kolmogorov length scale with data of Stanislas et al [1] and Herpin et al [3]. Herpin et al [3]: $\star, R e=10140 ; \bullet, R e=18950$. Other symbols as in figures 1 and 2 .

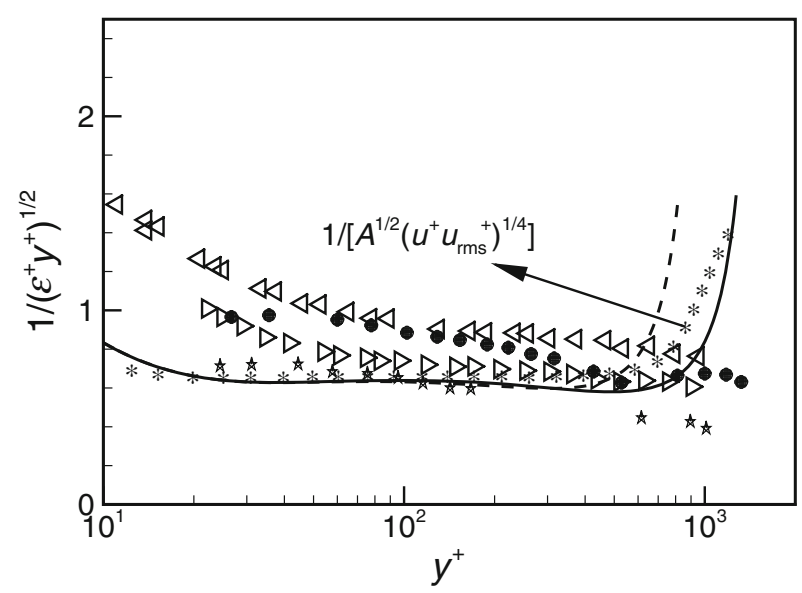

Figure 8. Scaling based on Kolmogorov time scale. Lines and symbols as in figures 1,2 and 7.

\section{Conclusion}

In summary, a model for the turbulent dissipation rate is proposed from a finding that $\left(u u_{\mathrm{rms}}\right) \sim u_{\tau}^{2}$ in the logarithmic region of a constant pressure boundary layer. This model is in terms of easily realizable mean flow quantities and can predict the turbulent dissipation rate over a large portion of the boundary layer, including the region of $\epsilon \approx$ constant. An alternative and easy means of experimentally measuring the Kolmogorov length scale and the Taylor microscale in the logarithmic region follows from this model for the turbulent dissipation rate.

\section{Acknowledgments}

The authors wish to thank Prof. J. K. Eaton for the data.

\section{References}

[1] Stanislas M, Perret L and Foucaut J M 2008 Vortical structures in the turbulent boundary layer: A possible route to a universal representation. J. Fluid Mech. 602: 327-382

[2] Segalini A, Örlü R, Schlatter P, Alfredsson P H, Rüedi J D and Talamelli A 2011 A method to estimate turbulence intensity and transverse Taylor microscale in turbulent flows from spatially averaged hot-wire data. Exp. Fluids 51: 693-700

[3] Herpin S, Stanislas M, Foucaut J M and Coudert S 2013 Influence of the Reynolds number on the vortical structures in the logarithmic region of turbulent boundary layers. J. Fluid Mech. 716: 5-50

[4] Pope S B 2000 Turbulent flows, 1st edn, Cambridge University Press, Cambridge.

[5] Blair M F and Bennett J C 1987 Hot-wire measurements of velocity and temperature fluctuations in a heated turbulent boundary layer. J. Phys. E Sci. Instrum. 20: 209-216

[6] Schlatter P and Örlü R 2010 Assessment of direct numerical simulation data of turbulent boundary layers. J. Fluid Mech. 659: $116-126$

[7] DeGraaff D B and Eaton J K 2000 Reynolds-number scaling of the flat-plate turbulent boundary layer. J. Fluid Mech. 422: 319-346

[8] Fukagata K, Iwamoto K and Kasagi N 2002 Contribution of Reynolds stress distribution to the skin friction in wallbounded flows. Phys. Fluids 14: L73

[9] Alfredsson P H, Örlü R and Segalini A 2012 A new formulation for the streamwise turbulence intensity distribution in wall-bounded turbulent flows. Eur. J. Mech. B-Fluids 36: $167-135$ 